\title{
Prognostic impact of $I K Z F 1$ deletions in association with vincristine-dexamethasone pulses during maintenance treatment of childhood acute lymphoblastic leukemia on trial ALL-BFM 95
}

Leukemia (2017) 31, 1840-1842; doi:10.1038/leu.2017.154

Acute lymphoblastic leukemia (ALL) is the most common malignancy of childhood and is cured by application of modern treatment protocols in more than $80 \%$ of cases. ${ }^{1,2}$ However, the $\sim 20 \%$ of children with ALL suffering from relapse still exemplify the ongoing need for improved risk stratification and adapted treatment strategies. ${ }^{1,2}$ In several studies, deletions of IKZF1encoding IKAROS, a transcription factor important for lymphoid development and differentiation-have been associated with a poor treatment outcome in precursor B cell ALL. ${ }^{3-5}$ Deletions of IKZF1 are observed in 10-15\% of pediatric ALLs and affect either the entire IKZF1 gene or appear as focal deletions. ${ }^{3-5}$ The most common of the latter ones $(\Delta 4-7)$ includes the DNA-binding region and results in a dominant-negative isoform (Ik6), impairing cell differentiation in CD34+ lymphoid progenitor cells. ${ }^{3-5}$

Recently, Clappier et al. ${ }^{6}$ from the EORTC Study Group suggested that patients with IKZF1-deleted ALL and intermediate-risk features-so-called average-risk (AR) patientstreated on the BFM-based EORTC protocol 58951 may benefit from intensification of conventional maintenance therapy by application of vincristine-glucocorticoid pulses. We previously reported that on ALL-BFM protocols IKZF1 status exerts additive value as a prognostic factor, especially in the intermediate-risk group, in which still the majority of relapses occur. ${ }^{4}$ Therefore, we investigated the impact of additional pulses during maintenance therapy for IKZF1-deleted ALL in a large cohort of intermediaterisk patients treated according to the ALL-BFM 95 protocol. ${ }^{7}$ These patients were included in the I-BFM study on pulses of vincristine and dexamethasone in BFM protocols for children with ALL. ${ }^{8}$

Between April 1995 and December 2000, patients diagnosed with de novo ALL were enrolled in ALL-BFM 95 from Austrian, German and Swiss study centers. ${ }^{7}$ Treatment was stratified in three risk groups as follows: standard (SR), intermediate (IR) and high risk (HR). Risk group definition was as follows: HR, prednisone poor response and/or no complete remission on day 33, and/or evidence of $\mathrm{t}(9 ; 22)$ (or $\mathrm{BCR} / \mathrm{ABL}$ ), and/or evidence of $\mathrm{t}(4 ; 11)$ (or MLL/AF4); IR, no HR criteria and initial white blood cell (WBC) count $20 \times 10^{9} /$ or more and/or age at diagnosis less than 1 or 6 years, or older, and/or T-ALL; SR, no HR criteria and initial WBC less than $20 \times 10^{9} / \mathrm{l}$, and age at diagnosis between 1 and 6 years, and no T-ALL. CNS status was no stratification criterion. Before start of maintenance treatment, patients of the IR group who were in complete remission were randomly assigned to receive either conventional mercaptopurine and methothrexate chemotherapy or mercaptopurine and methothrexate supplemented with additional pulses of vincristine $\left(1.5 \mathrm{mg} / \mathrm{m}^{2}\right.$ weekly for 2 weeks) and dexamethasone $\left(6 \mathrm{mg} / \mathrm{m}^{2}\right.$ daily for 7 days) every 10 weeks for six cycles. IKZF1 deletion status in leukemic DNA isolated from initial bone marrow smears was assessed by a multiplex PCR assay ${ }^{9}$ (Supplementary Figure 1).

We were able to isolate leukemic DNA from bone marrow smears of 655 German precursor B cell ALL patients-corresponding to $95 \%$ of the entire randomized German cohort of the patients who were treated as randomized-who were subsequently subjected to PCR screening for IKZF1 deletion status. ${ }^{9}$ Analyzed patients did not differ from those not included due to lack of biological specimens (Supplementary Table 1). Characteristics of IR patients were comparable in the two randomized arms (Supplementary Table 2). In accordance with previously reported results of the I-BFM study on pulses, ${ }^{8}$ no differences in outcome were observed between the experimental and standard treatment arms (Supplementary Figure 2). Out of the 655 patients included in our study, 80 tested positive for an IKZF1 deletion (12.2\%). In analyses of the entire group, IKZF1 deletion was associated with a worse treatment outcome compared to IKZF1 wild-type status (5year event-free survival (EFS): $0.66 \pm 0.05$ vs $0.82 \pm 0.02, P=0.001$; Supplementary Figure 3). When outcome was analyzed restricted to IKZF1-deleted patients, 5-year EFS tended to be lower in patients treated on the experimental arm incorporating pulses compared to those on standard therapy $(0.57 \pm 0.08$ vs $0.74 \pm 0.07$, $P=0.083$ ) (Figure 1a). Non-relapse events were more frequent in the experimental arm and contributed to the differences observed in EFS analyses (Supplementary Table 3). Thirteen (35.1\%) patients exposed to experimental treatment suffered from relapse compared to $11(25.6 \%)$ patients on the standard arm; respective cumulative relapse incidences (CIR) were not significantly different $(0.35 \pm 0.08$ vs $0.26 \pm 0.07 ; P=0.370$; Figure $1 \mathrm{~b})$. In multivariate analysis within the IR group, IKZF1 deletion was significantly associated with risk of an event only in patients receiving pulsed maintenance treatment, while this effect was not observed for IKZF1-deleted patients on the standard arm (Table 1). However, as mentioned already above, the significant hazard ratio for IKFZ1 deletion on the experimental arm is likely to be explained by the higher number of non-relapse events observed in this group (Supplementary Table 3). Thus, application of pulsed maintenance therapy did not specifically improve outcome for IKZF1-deleted IR patients on ALL-BFM 95.

For 34 (15.5\%) IKZF1-deleted out of 219 randomized AR patients on EORTC trial 58951, Clappier and colleagues described an outcome for IKZF1-deleted patients who received pulses, which was identical to that of non-deleted patients (8-year DFS: 93.3 versus $89.5 \% ; P=0.600$ ), whereas the outcome for deleted patients who did not receive pulses was significantly worse compared to IKZF1 wild-type patients (8-year DFS 42.1 versus $88.8 \%$; $\mathrm{HR}=6.65$; $P<0.001) .{ }^{6}$ Consequently, the authors suggested that intensification of maintenance therapy with vincristine-glucocorticoid pulses had contributed to prevent relapses in patients with deletions of IKZF1. Treatment on both trials ALL-BFM 95 and EORTC trial 58951 was BFM-based and comparable, as was overall 
IR and AR outcome with 6-year DFS of 83.2 and $82.2 \%$, respectively. However, potential explanations for the observed differences in treatment results for IKZF1-deleted patients receiving pulsed maintenance therapy on these two trials include, for example, differences in risk group definition. IR patients on ALLBFM 95 were aged less than 1 or 6 years or older, or had initial
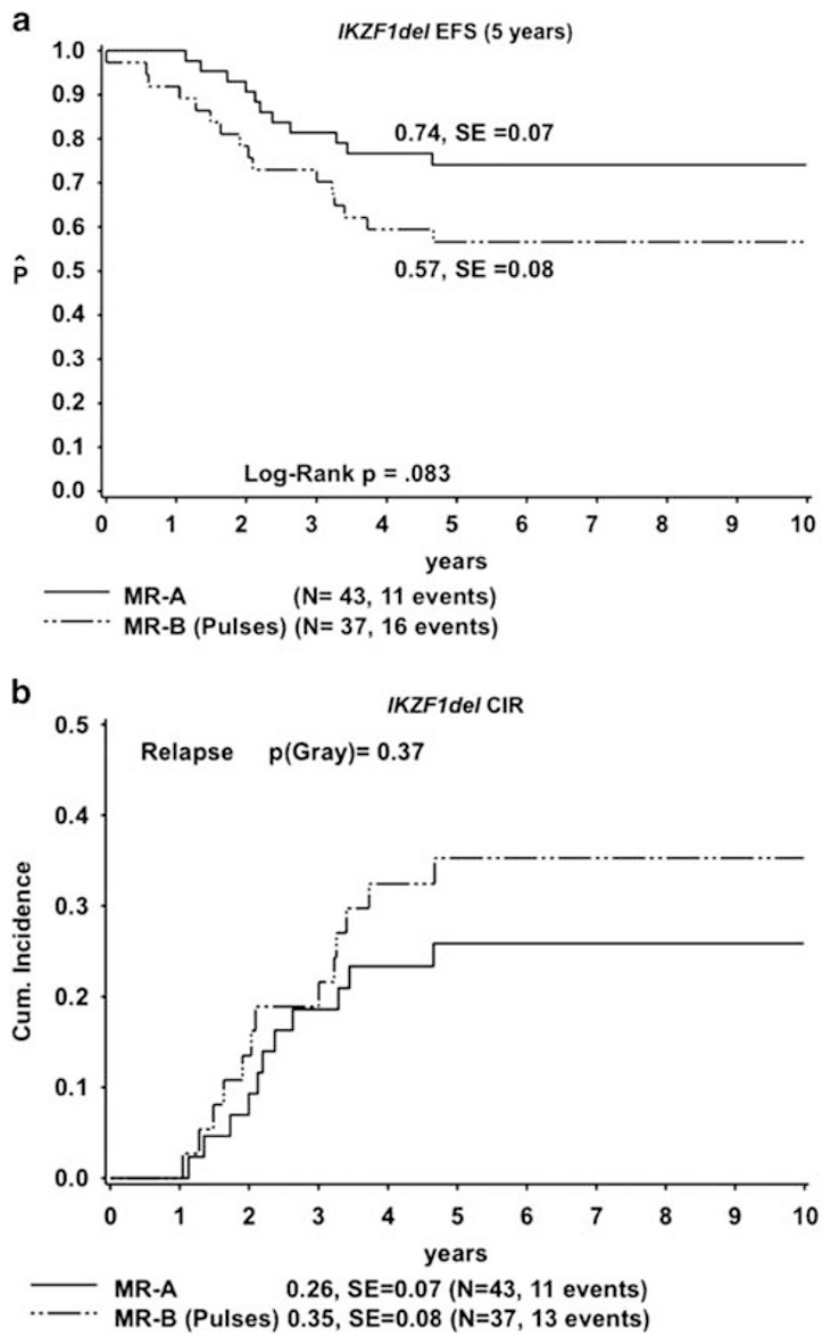

Figure 1. Treatment outcome of IKZF1 deletion-positive patients by conventional and pulsed maintenance therapy. Kaplan-Meier estimates of 5-year event survival (a) and cumulative incidences of relapse (b) are shown.
WBC counts of $20 \times 10^{9} /$ or more, had a favorable cytomorphologically assessed treatment response and were negative for a $\mathrm{t}(4 ; 11)$ or its molecular equivalent MLL-AF4. Minimal residual disease (MRD) analyses were not performed on ALL-BFM 95. On EORTC 58951, AR patients were not restricted to certain age groups, had initial WBC counts of $10 \times 10^{9} /$ or more, had a favorable cytomorphologically assessed treatment response, were not only negative for all MLL rearrangements, but also for high hyperdiploidy (51 or more chromosomes or DNA index $>1.16$ and $<1.50$ ) as well as low hypodiploidy or near haploidy. In addition, the EORTC trial excluded patients with high MRD levels $\left(10^{-2}\right.$ or more) after induction treatment from the AR group. Therefore, the stratification strategy on ALL-BFM 95 may have selected for a different spectrum of IKZF1-deleted ALL patients in our study in comparison to the EORTC study. To get an estimate on the percentage of patients, which would have been excluded from the IR group when MRD-PCR analyses would have been conducted, we applied the ALL-BFM 95 stratification criteria to patients from trial ALL-BFM 2000, which used DNA-PCR-based MRD analyses for stratification. ${ }^{4,10}$ Of 2386 ALL-BFM 2000 patients fulfilling the ALLBFM 95 IR stratification criteria and having the MRD data available, only $159(6.7 \%)$ would have been stratified into the HR group according to the level of $\geqslant 10^{-2}$ as applied in the EORTC 58951 trial (Supplementary Table 4), which could have added to the differences observed between the two studies. One additional indicator for differences in patient populations could be the fact that the relapse cascade on ALL-BFM 95 started already during maintenance treatment, while on EORTC 58951 the majority of relapses was observed after cessation of treatment. If we look at the relapse pattern of patients from ALL-BFM 2000 with the available MRD data but stratified according to the ALL-BFM 95 criteria, we observe a similar relapse cascade for these "ALL-BFM 95 IR patients" who would have been stratified to HR on ALL-BFM 2000 according to their MRD levels. ${ }^{10}$ As treatment approaches between the ALL-BFM 95 IR and EORTC 58951 AR groups were comparable, this suggests that the stratification strategy on trial EORTC 58951 was more effective in preventing early relapsesprobably through exclusion of patients with MRD levels of $\geqslant 10^{-2}$ after induction to the HR group.

Lastly, our diagnostic approach itself could have influenced results through selective exclusion of patients affected by whole IKZF1 gene deletions. ${ }^{9}$ We are not aware of the distribution of these patients in our study cohort, which could have led to bias in our assessment. However, assuming a balanced distribution in the two randomized groups-as observed for focal deletions detected by our multiplex PCR approach-the probability of a severe influence on our results seems negligible.

In conclusion, our data demonstrate that intensification of maintenance therapy with vincristine-glucocorticoid pulses in IR patients with IKZF1-deleted ALL is not associated with improved outcome on trial ALL-BFM 95.

\begin{tabular}{|c|c|c|c|c|c|c|}
\hline & \multicolumn{3}{|c|}{ Conventional maintenance therapy } & \multicolumn{3}{|c|}{ pulsed maintenance therapy } \\
\hline & Hazard ratio & 95\% confidence interval & P-value & Hazard ratio & 95\% confidence interval & P-value \\
\hline IKZF1 deletion ${ }^{\mathrm{a}}$ & 1.32 & $0.69-2.52$ & 0.403 & 2.97 & $1.67-5.29$ & $<0.001$ \\
\hline WBC count at diagnosis $\geqslant 100000 /\left.\mu\right|^{\mathrm{b}}$ & 3.12 & $1.54-6.31$ & 0.002 & 1.41 & $0.60-3.33$ & 0.431 \\
\hline Sex ${ }^{c}$ & 0.65 & $0.41-1.05$ & 0.078 & 0.80 & $0.48-1.34$ & 0.420 \\
\hline Age at diagnosis $\geqslant 10$ years $^{d}$ & 1.35 & $0.82-2.22$ & 0.243 & 1.59 & $0.94-2.69$ & 0.087 \\
\hline
\end{tabular}

Abbreviations: ALL, acute lymphoblastic leukemia; WBC, white blood cell. ${ }^{a}$ In comparison to negative patients. ${ }^{b}$ In comparison to WBC count at diagnosis $<100000 / \mu$ l. ' Females compared to male patients. ${ }^{d}$ In comparison to patients $<10$ years. 
1842

\section{CONFLICT OF INTEREST}

The authors declare no conflict of interest.

\section{ACKNOWLEDGEMENTS}

We are grateful to all patients and colleagues participating in trial ALL-BFM 95. Supported by the European commission under the 7th Framework Programme (FP7; ERA-NET grant TRANSCALL), Madeleine-Schickedanz-Kinderkrebsstiftung, Deutsche Krebshilfe, Verein für krebskranke Kinder Hannover e.V.

L Hinze ${ }^{1}$, A Möricke ${ }^{2}$, M Zimmermann ${ }^{1}$, S Junk ${ }^{1}$, G Cario ${ }^{2}$, E Dagdan ${ }^{1}, \mathrm{CP} \mathrm{Kratz}^{1}$, V Conter ${ }^{3}, \mathrm{M} \mathrm{Schrappe}^{2}$ and M Stanulla ${ }^{1}$

${ }^{1}$ Department of Pediatric Hematology and Oncology, Hannover Medical School, Hannover, Germany;

${ }^{2}$ Department of Pediatrics, University Hospital Schleswig-Holstein, Kiel, Germany and

${ }^{3}$ San Gerardo Hospital, Pediatric Clinic, Monza, Italy E-mail: stanulla.martin@mh-hannover.de

\section{REFERENCES}

1 Vrooman LM, Silverman LB. Treatment of childhood acute lymphoblastic leukemia: prognostic factors and clinical advances. Curr Hematol Malig Rep 2016; 11: 385-394.

2 Moorman AV. New and emerging prognostic and predictive genetic biomarkers in B-cell precursor acute lymphoblastic leukemia. Haematologica 2016; 101: 407-416.

3 Mullighan CG, Su X, Zhang J, Radtke I, Phillips LAA, Miller CB et al. Deletion of IKZF1 and prognosis in acute lymphoblastic leukemia. N Engl J Med 2009; 360: 470-480.

4 Dörge P, Meissner B, Zimmermann M, Möricke A, Schrauder A, Bourqin J-P et al. IKZF1 deletion is an independent predictor of outcome in pediatric acute lymphoblastic leukemia treated according to the ALL-BFM 2000 protocol. Haematologica 2013; 98: 428-432.
5 van der Veer A, Zaliova M, Mottadelli F, De Lorenzo P, te Kronnie G, Harrison C-J et al. IKZF1 status as a prognostic feature in BCR-ABL1-positive childhood ALL. Blood 2014; 23 (11): 1691-1698.

6 Clappier E, Grardel N, Bakkus M, Rapion J, De Moerloose B, Kastner P et al. IKZF1 deletion is an independent prognostic marker in childhood B-cell precursor acute lymphoblastic leukemia, and distinguishes patients benefiting from pulses during maintenance therapy: results of the EORTC Children's Leukemia Group study 58951. Leukemia 2015; 29: 2154-2161.

7 Möricke A, Reiter A, Zimmermann M, Gadner H, Stanulla M, Dördelmann M et al. Risk-adjusted therapy of acute lymhoblastic leukemia can decrease treatment burden and improve survival: treatment results of 2169 unselected pediatric and adolescent patients enrolled in the trial ALL-BFM 95. Blood 2009; 113: 4478

8 Conter V, Valsecchi MG, Campbell M, Dibar E, Magyarosy E, Gadner H et al. Pulses of vincristine and dexamethasone in addition to intensive chemotherapy for children with intermediate risk acute lymphoblastic leukaemia: a multicentre randomised trial. Lancet 2007; 369: 123-131.

9 Caye A, Beldjord K, Mass-Malo K, Drunat S, Soulier J, Gandemer V et al. Breakpointspecific multiplex polymerase chain reaction allows the detection of IKZFI intragenic deletions and minimal residual disease monitoring in B-cell acute lymphoblastic leukemia. Haematologica 2013; 98: 597-601.

10 Conter V, Bartram CR, Valsecchi MG, Schrauder A, Panzer-Grümayer R, Möricke $A$ et al. Molecular response to treatment redefines all prognostic factors in children and adolescents with B-cell precursor acute lymphoblastic leukemia: results in 3184 patients of the AIEOP-BFM ALL 2000 study. Blood 2010; 115: 3206-3214.

(C) This work is licensed under a Creative Commons Attributioncc. NonCommercial-ShareAlike 4.0 International License. The images or other third party material in this article are included in the article's Creative Commons license, unless indicated otherwise in the credit line; if the material is not included under the Creative Commons license, users will need to obtain permission from the license holder to reproduce the material. To view a copy of this license, visit http:// creativecommons.org/licenses/by-nc-sa/4.0/

(c) The Author(s) 2017

Supplementary Information accompanies this paper on the Leukemia website (http://www.nature.com/leu) 